\title{
ALTERNATIVE RELATIVISTIC FORMULATION OF LINEAR SPECTRUM OF HYDROGEN-LIKE SYSTEMS
}

\author{
Ilya E. Eremin \\ Department of Mathematic and Informatics \\ Amur State University \\ Russia \\ ilya.eremin.70@mail.ru
}

\author{
Vitaliy V. Neshtimenko \\ Engineering-Physical Department \\ Amur State University \\ Russia \\ v1ta1y@mail.ru
}

\author{
Dmitry S. Shcherban \\ Department of Mathematic and Informatics \\ Amur State University \\ Russia \\ dmitriy1987@bk.ru
}

\author{
Denis V. Fomin \\ Department of Mathematic and Informatics \\ Amur State University \\ Russia \\ gefest-uni@yandex.ru
}

Article history:

Received 30.08.2020, Accepted 20.12.2020

\begin{abstract}
The article describes the possibility of describing the electronic structure of the simplest atomic systems within corpuscular physics theory. The proposed method allows to derive the Rydberg frequency constant without using quantum Bohr postulates. Adequate results of the same type of calculation of the energy levels of the first five chemical elements that are in the maximum degree of ionization are presented.
\end{abstract}

\section{Key words}

Quantum transitions, relativistic effects, frequency resonances, Control of oscillations, Modeling, Quantum control

\section{Introduction}

At present, it is considered that the most effective way to describe the electronic properties of a matherial is a discrete-stochastic modelling of the spectral picture of the energy characteristics of the matherial's particles, implemented using methods of modern quantum mechanical calculations [Miao et al., 2014; Sych and Leuchs, 2015; Ogata, 2016; Nakajima and Tokura, 2017; Han, Xu and Qin, 2018]. Along with this, there is another opinion openly expressed by many well-known scientists, for example, Professor N. Kobayashi of the University of Tokyo: "This task is extremely difficult, because the existing methods of modeling atomic and molecular structures are imperfect and require too much time. Progress in solving the task can be achieved both by improving the numerical methods of direct calculations and increasing their reliability, and by developing completely new methods based on completely different principles of computation" [Kobaysi, 2007].

As already known, any utilitarian method of quantum calculations, one way or another, uses the principle of complementarity, which follows from the inevitability of quantum jumps within a certain set of electronic levels, initially postulated by N. Bohr [Roldan-Charria, 2014; Robinson and Haven, 2015; Hansen, 2016]. The key confirmation of the validity of this interpretation is the theoretical justification within its framework of the Rydberg frequency constant. In turn, the most vulnerable point of the atom's structure model of Bohr is due to the criticism of the mathematical induction of quantum and classical principals performed by him for the purpose of correct description of actually observed linear spectrums. E. Rutherford also pointed out: "I think your hypothesis has another very weak point. I have no doubt that yourself are fully aware of this. How does an electron decide at what frequency it will oscillate when moving from one stationary state to another?" [Kumar, 2013].

However, Bohr and his supporters persistently insisted on the absolute theoretical completeness of the probabilistic interpretation of quantum mechanics developed by them, the completeness of which, in turn, was questioned by A. Einstein, who was confident in the existence of a deeper physical level, including additional, temporarily hidden information that strictly determines the true laws of the quantum world. In other words, in 
modern quantum theory, there is a situation of a classical "black box", the input and output parameters of which are well-known, and there is no explicit physical and mathematical description of the mechanism of their cause-and-effect relationship at the current time, one way or another.

Thus, the problem of complex re-evaluation of inductive propositions of classical quantum mechanics, implemented in the deductive framework of a systematic approach, whose initial potential should help to eliminate the key shortcomings of quantum mechanical calculations, as well as simplify the mathematical synthesis of effective models of electronic properties and structure of specific substances, becomes particularly relevant [Kostyukov and Eremin, 2004; Kostyukov and Eremin, 2008; Eremin et al., 2010; Eremin et al., 2014].

The alternative interpretations proposed in this paper are based on the deductive method of analyzing the internal structure and resulting properties of the systems under study. Such a revision of the classical models of the objects under study from the standpoint of the system approach is necessary for an objective assessment of the possibility of their effective use in the search for algorithms for controlling certain processes. Because really effective management is possible only with a sufficient level of understanding of the control object.

These interpretations give a qualitatively different picture of the location of electronic orbitals in space, i.e. they completely change the worldview of the original quantum object and can potentially influence the choice of certain ways to control its properties.

By the authors' opinion the alternative relativistic formulation of the linear spectra of hydrogen-like systems and the alternative model of the electronic levels of the hydrogen atom presented in this paper can increase the accuracy and reduce the computational complexity of models of quantum processes and phenomena. This, in turn, will allow us to develop more efficient models and algorithms for controlling such systems, which is one of the tasks solved within such sections of Cybernetics as quantum control, oscillation control and optimal control [Fradkov, 2017; Rabitz, 2012; James, 2005; Doherty, 2000; Dong, 2010; D’Alessandro, 2008; Averbukh, 2016; Jacobs, 2014; Youssry, 2020].

\section{Classical Discrete-Stochastic Model}

In the middle of the XIX century, it was experimentally discovered that the passage of a light beam through a gas located in the zone of action of an electrostatic discharge leads to forming of a unique set of narrow spectral lines that uniquely characterize the substance under study. At the same time, a series of spectral lines of highly rarefied hydrogen, physically observed in the red, green, blue and violet sections of the optical spectrum's visible range, attracted particular attention of scientists.

The wavelengths' numerical values of the electromagnetic field of light corresponding to the considered linear spectrum were measured in 1868 by A. Angstrom and amounted to $656.21,486.07,434.01$ and $410.12 \mathrm{~nm}$ respectively. In 1884, I. Balmer managed to find an empirical formula that adequately describes the wavelength of each listed line:

$$
\lambda=\frac{b m^{2}}{m^{2}-n^{2}},
$$

where $n$ and $m$ - natural numbers $(n=2 ; m=3,4,5$, $6) ; b$ - the longwave Balmer constant equals $364.56 \mathrm{~nm}$.

In $1890 \mathrm{~J}$. Rydberg derived a generalized equation that allows us to calculate the frequencies of all theoretically possible spectral lines of hydrogen:

$$
f_{r}=\left(\frac{1}{n^{2}}-\frac{1}{m^{2}}\right) R,
$$

where $R$ - Rydberg's frequency constant, equals $3.289842 \cdot 10^{15} \mathrm{~s}^{-1} ; n$ - natural number, indicating sequential number of a series; $m$ - natural number that start with the value $n+1$ and characterize all lines of the $n$-th series.

It should be noted that the spectral formula of the general form (2) was initially derived empirically and for a long time did not have an acceptable theoretical justification, although it was confirmed experimentally with a very high accuracy. However, the striking repeatability of natural numbers in it and the objective universality of the Rydberg constant very evidently indicate a deep physical meaning of the laws of the electron-atomic structure of matter detected with its help, an abstract description of which is considered fundamentally impossible within the framework of the traditional (inductive) approach of classical physics.

The first attempt to build a qualitatively new (quantum) theory of the structure of the atom was made in 1913 by N. Bohr. It was based on two postulates that combined the following set of fundamental assumptions accumulated by that time. First - the regularity of the linear hydrogen spectra revealed by Balmer and Rydberg. Secondly, the quantum nature of light emission and absorption discovered in 1900 by M. Planck and theoretically justified in 1905 by A. Einstein. And the planetary model of the atom, proposed in 1911 by E. Rutherford.

The Bohr's first postulate regulates that atoms have stable states in which it does not emit energy. In this case, they correspond to stationary electronic orbits, moving along which the electron must have discrete values of the moment of momentum that satisfy the condition:

$$
m_{e} v_{n} r_{n}=n \hbar,
$$

where $m_{e}$ - the rest mass of the electron, equal to $9.109543 \cdot 10^{-31} \mathrm{~kg} ; v_{n}$ - its linear velocity of circular motion along the $n$-th orbit; $r_{n}$ - the radius of the electron orbit; $\hbar$ - the Dirac constant, equal to 1.054572 . $10^{-34} J \cdot s(2 \pi \hbar=h, h-$ the quantum Planck constant, equal to $6.625 \cdot 10^{-34} \mathrm{~J} \cdot \mathrm{s}$ ). 
The Bohr's second postulate states that when an electron passes from one stationary orbit to another, a photon is emitted or absorbed with an energy equal to the energy difference of the appropriate electronic levels:

$$
h f_{r}=E_{\text {out }}-E_{\text {in }},
$$

where $E_{\text {out }}$ and $E_{\text {in }}$ - are the energies corresponding to the initial and final orbits. Based on such a hypothetical interpretation, for the case of $E_{\text {out }}>E_{\text {in }}$ an atom emits a light photon; if $E_{\text {out }}<E_{\text {in }}$, the opposite situation is observed, which is related to the atomic absorption of a quantum of light [Bohr, 1913].

Thus, the initial theoretical assumptions postulated by Bohr made it possible to mathematically express two physical constants - the value of the first Bohr radius and the Rydberg frequency constant. At the same time, a planetary model of a hydrogen-like Rutherford's atom was used for their conclusion, in which the force of the Coulomb interaction of elementary particles gives the rotating electron a certain centripetal acceleration, i.e. the basic law of the system dynamics has the form:

$$
\frac{Z e^{2}}{4 \pi \varepsilon_{0} r_{n}^{2}}=\frac{m_{e} v_{n}^{2}}{r_{n}}
$$

where $Z$ - the sequence number of the atom; $e$ - the elementary charge equal to $1.602189 \cdot 10^{-19} C$; $\varepsilon_{0}$ - the permittivity of the vacuum, equal to $8.854188 \cdot 10^{-12} \mathrm{~F} / \mathrm{m}$.

On the one hand, using expression (5), systemically combined with expression (3), Bohr derived a theoretical formula for the radiuses of stationary electron orbits of a hydrogen-like system:

$$
r_{n}=r_{B} \frac{n^{2}}{Z}, r_{B}=\frac{4 \pi \varepsilon_{0} \hbar^{2}}{m_{e} e^{2}},
$$

here $r_{B}$ - the value of the first Bohr radius, hypothetically representing the electron orbit of the hydrogen atom and equal to $0.529177 \cdot 10^{-10} \mathrm{~m}$.

On the other hand, taking into account that the total energy $E_{n}$ of an electron evenly rotating around a stationary atomic nucleus in the $n$-th orbit consists of its kinetic energy and the potential energy of the Coulomb interaction of elementary particles, Bohr formed the expression:

$$
E_{n}=\frac{m_{e} v_{n}^{2}}{2}-\frac{Z e^{2}}{4 \pi \varepsilon_{0} r_{n}} .
$$

The system combination of relations (5)-(7) gave the result:

$$
E_{n}=-\left(\frac{Z^{2}}{n^{2}}\right) \frac{1}{2} \frac{m_{e} e^{4}}{\left(4 \pi \varepsilon_{0}\right)^{2} \hbar^{2}},
$$

based on this, it was concluded that the energy states of the atom are a sequence of electronic levels, given by their numbers $n$ (the main quantum numbers). In this case, the first level is the main (normal) state, and all the others are excited.
By assigning the appropriate values to the number $n$, it became possible to obtain a hypothetical image of the stationary orbits of the hydrogen atom, as well as to form a scheme of potential transitions between them (Figure 1).
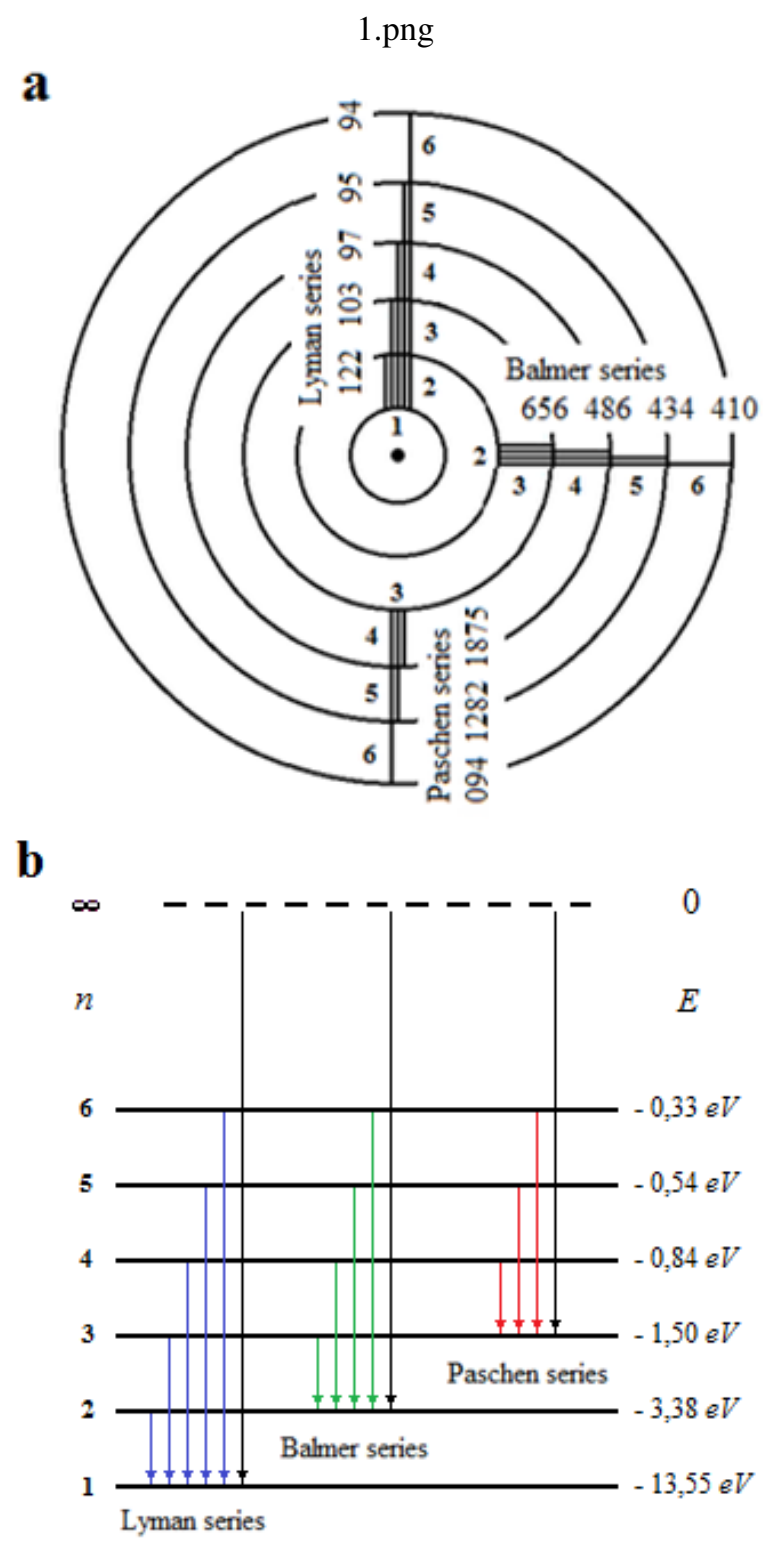

Figure 1. Bohr's interpretation of the electron levels of the hydrogen atom. a) diagram of the locations of stationary electronic orbits. b) schematic graph of the hypothetical quantum jumps that regulate the induction of linear spectral series of Lyman, Balmer, and Paschen.

In turn, based on the second Bohr's postulate (4), the transition of a hydrogen atom (i.e. for the case $Z=1$ ) from a certain excited state $m$ to the stable state $n$ emits an energy quantum

$$
h f_{r}=-\left(\frac{1}{m^{2}}-\frac{1}{n^{2}}\right) \frac{m_{e} e^{4}}{2\left(4 \pi \varepsilon_{0}\right)^{2} \hbar^{2}},
$$


therefore, the physically observed frequency of the corresponding light radiation can be represented as:

$$
f_{r}=\left(\frac{1}{n^{2}}-\frac{1}{m^{2}}\right) \frac{m_{e} e^{4}}{2\left(4 \pi \varepsilon_{0}\right)^{2} \hbar^{2} h} .
$$

By comparing the empirical spectral formula (2) with his theoretical equation (10), Bohr obtained an expression that fully corresponds to the experimentally measured value of the Rydberg frequency constant [Bohr, 1913]:

$$
R=\frac{m_{e} e^{4}}{2\left(4 \pi \varepsilon_{0}\right)^{2} \hbar^{2} h}=\frac{m_{e} e^{4}}{8 h^{3} \varepsilon_{0}^{2}} .
$$

It is generally recognized that Bohr's description of the electron levels of hydrogen was a significant step in the development of atomic physics and laid the Foundation for the modern quantum mechanics, introducing a worldview picture of a series of quantum jumps into scientific circulation. However, Bohr's theory could not explain the physical reasons for the implementation of certain electronic transitions, as well as the intensity of the corresponding spectral lines. In addition, its significant disadvantage was the inability to adequately describe the linear spectrum of the helium atom directly following the hydrogen.

In 1923, L. de Broglie hypothesized that any atomic structures independently possess both corpuscular and wave properties. In turn, experimental confirmation of the general universality of the wave-particle duality, as well as the limited application of classical mechanics for an adequate description of objects in the microworld led to occurrence of the quantum mechanics, based on the theoretical research of V. Heisenberg (matrix mechanics) and E. Schrodinger (wave mechanics), as well as the probabilistic interpretation of the wave function of $\mathrm{M}$. Born.

Thus, modern methods of quantum calculations, which are being formed or improved over the past decades, are based on the "Copenhagen concept", which includes: the Bohr correspondence principle, the Heisenberg uncertainty principle, the probabilistic interpretation of the Schrodinger-Born's wave function, the Broglie-Bohr's complementarity principle, and the collapse of the wave function. Note that N. Bohr and his supporters insisted on the scientific completeness of the named concept, which was doubted by A. Einstein, confident in the existence of some "hidden parameters" that determine quantum effects at a deeper level.

\section{Energy Diagrams of an Electronic Oscillator}

At the end of the nineteenth century, it was discovered that the laws of the Newtonian mechanics contradict experimental data obtained in the study of the movement of fast elementary particles. In addition, it was found that the existing theory of electricity diverged from the equations of J. Maxwell, underlying the understanding of light as an electromagnetic wave. In other words, it became necessary to search for a paradigm that, explaining these facts, would include the Newton's dynamics as the limiting case of describing small speeds. In 1905, A. Einstein developed the basic tenets of the special theory of relativity (relativistic theory), based on two postulates introduced by him - the principle of relativity, which asserts the invariance of the laws of nature with respect to the transition between inertial reference systems, and the principle of invariance of the speed of light.

Taking into account relativistic effects that objectively occur in any high-speed systems, the physically observed mass of an electron $m^{\prime}$, as well as the experimentally measured distance between it and the atomic nucleus $r^{\prime}$, should be expressed by the Lorentz-Einstein's equations, at least in the context of modeling the linear spectra of hydrogen-like systems:

$$
m^{\prime}=\frac{m_{e}}{\sqrt{1-v^{2} / c^{2}}} ; r^{\prime}=\frac{r}{\sqrt{1-v^{2} / c^{2}}},
$$

here $m_{e}$ - the rest mass of an electron; $r$ - the true radius of its orbit; $v$ - the real velocity of the electron; $c$ - the speed of light in a vacuum, equal to $299792458 \mathrm{~m} / \mathrm{s}$.

Therefore, the normal component of the kinetic energy $T_{e}$ of an electron, determined by the centrifugal force $F_{\text {rot }}$ resulting from its circular motion, can be represented as follows:

$$
\begin{gathered}
F_{r o t}=\frac{m^{\prime} v^{2}}{r^{\prime}}=\frac{m_{e} v^{2}}{r} ; \\
T_{e}=F_{r o t} r \Rightarrow T_{e}=m_{e} v^{2} .
\end{gathered}
$$

In turn, the potential energy $P_{e}$ of a rapidly rotating electron, due to the strength of its electrostatic (Coulomb) interaction $F_{k u l}$ with the atomic nucleus, can be expressed as follows:

$$
\begin{gathered}
F_{k u l}=\frac{Z e^{2}}{4 \pi \varepsilon_{0} r^{\prime 2}}=\frac{Z e^{2}\left(1-v^{2} / c^{2}\right)}{4 \pi \varepsilon_{0} r^{2}} ; \\
P_{e}=F_{k u l} r \Rightarrow P_{e}=\frac{Z e^{2}}{4 \pi \varepsilon_{0} r}\left(1-\frac{v^{2}}{c^{2}}\right) .
\end{gathered}
$$

It is quite obvious that the stereotypical form of the function (16) considered relatively to the distance between the electron and the atomic nucleus is a simple hyperbolic graph. On the other hand, to increase its general informativeness, i.e., to display the dependence of $P_{e}(r)$, simultaneously characterized by the current electron speed, the authors suggest using the theoretical scheme of the "planetary converter" (Figure 2).

In other words, there is a completely adequate corpuscular model, which has the following formulation. First, an electron located on the surface of an atomic 

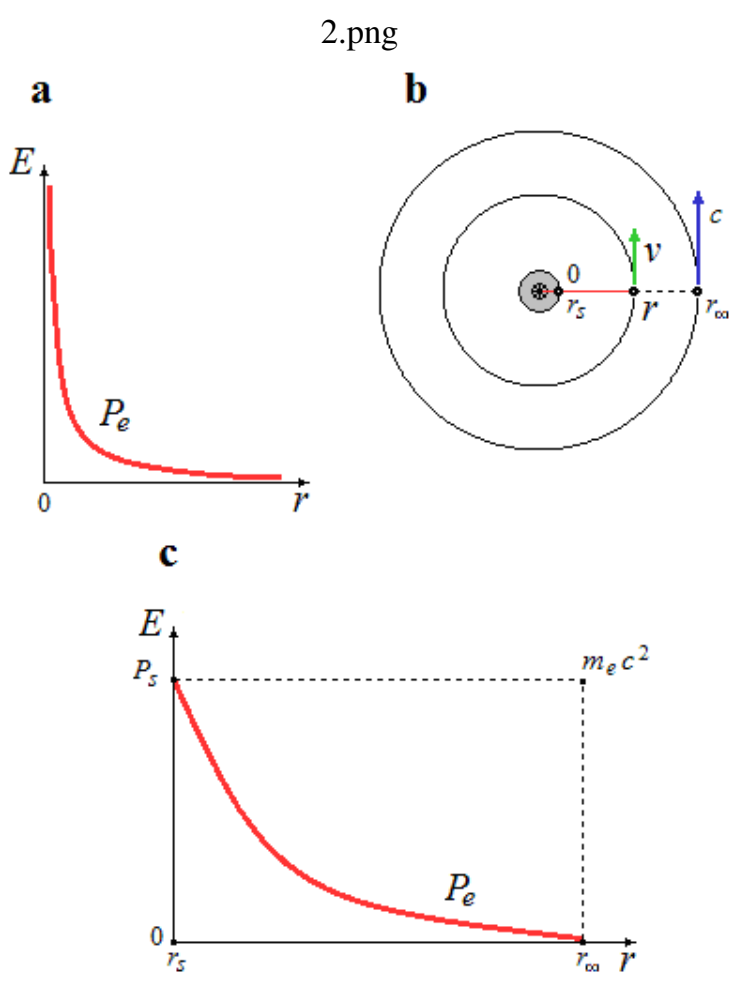

Figure 2. Visualization of mathematical models of the potential energy of an electron. a) graph of a function of the general form (16). b) theoretical scheme of the "planetary converter". c) graph of the function $P_{e}(r)$ model-fixed with respect to the current electron velocity.

nucleus of radius $r_{s}$ is stationary in its own (atomic) frame of reference and has the highest potential energy: $P_{s}=Z e^{2} /\left(4 \pi \varepsilon_{0} r_{s}\right)$. Second, an electron moving evenly along a certain stable circular orbit of radius $r$ has a well-defined linear velocity $v$. Third, when the electron orbit's radius increases to infinitely long distance $r_{\infty}$, the corresponding value $v$ becomes equivalent to the speed of light, and the kinetic energy of the electron, estimated in the global reference system, is extremely permissible: $T_{\infty}=m_{e} c^{2}$.

In addition, the electronic spectrum of the studied systems is effectively described by the Planck's quantum ratio, which can be related to the above-mentioned atomic parameters $r$ and $v$ :

$$
\begin{gathered}
E=n h f ; \\
f=\frac{v}{2 \pi r} \Rightarrow E=n h \frac{v}{2 \pi r} .
\end{gathered}
$$

It is easy to see that equations (16) and (18) use different physical quantities. The potential energy $P_{e}$ is expressed in terms of the velocity ratio $v^{2} / c^{2}$, and the Planck's energy $E$ takes into account the main quantum number $n$. The authors of the article believe that this discrepancy can be eliminated by parametric substitution, which does not contradict the concept of quantization in principle:

$$
\frac{v}{c} \rightarrow \frac{n}{m} \Rightarrow \sqrt{1-\frac{v^{2}}{c^{2}}}=\sqrt{1-\frac{n^{2}}{m^{2}}} .
$$

Thus, the dependency expression $P_{e}(r)$ is converted to the form:

$$
P_{e}=\frac{Z e^{2}}{4 \pi \varepsilon_{0} r}\left(1-\frac{n^{2}}{m^{2}}\right)
$$

In turn, taking into account the model-fixed graph $P_{e}(r)$, as well as the law of conservation of energy, it becomes possible to construct diagrams of the total energy of an elementary oscillator (Figure 3).

\section{3.png}

a

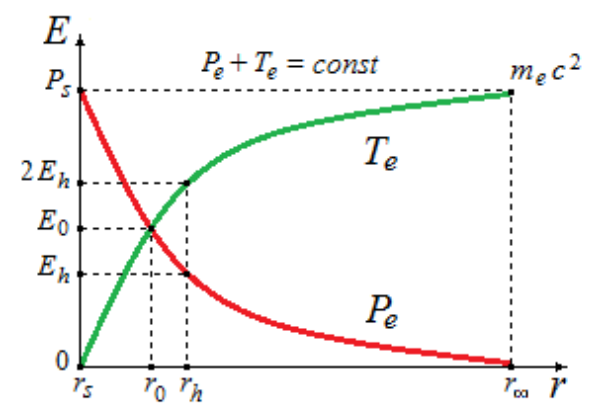

b

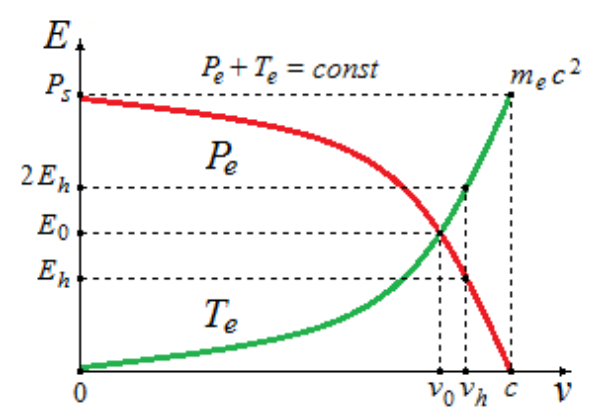

Figure 3. Energy diagrams of an electronic oscillator. a) graphs of energy functions schematized with regard to the distance between the electron and the atomic nucleus. b) graphs of energy functions schematized with regard to the the linear velocity of the circular motion of the electron around the nucleus.

The resulting scheme makes clear the situation $T_{e}\left(v_{0}\right)=E\left(r_{0}, v_{0}\right)=P_{e}\left(r_{0}\right)$, which is characteristic of the value of the electron's first orbital velocity $v_{0}$ in the case of its even movement along the minimum stable orbit of radius $r_{0}$. In this case, the Planck's relation (17), considered in the form (18), can be used to give argumentative uniformity to functions (14) and (20).

Indeed, the first part of the considered equilibrium condition for a hydrogen-like system $T_{e}\left(v_{0}\right)=E\left(r_{0}, v_{0}\right)$, i.e. an expression:

$$
m_{e} v_{0}^{2}=n h \frac{v_{0}}{2 \pi r_{0}}
$$


allows to get the following intermediate result:

$$
v_{0}=\frac{n h}{2 \pi r_{0} m_{e}} .
$$

In turn, the key component of the condition, converted to the form $T_{e}\left(r_{0}\right)=P_{e}\left(r_{0}\right)$ with consideration of the expression (22), i.e. the relation of the form:

$$
m_{e}\left(\frac{n h}{2 \pi r_{0} m_{e}}\right)^{2}=\frac{Z e^{2}}{4 \pi \varepsilon_{0} r_{0}}\left(1-\frac{n^{2}}{m^{2}}\right) ;
$$

directly generates a formula for the radial values for the entire set of stationary states, reasoned by the quantum parameters $n$ and $m$ :

$$
r_{0}(n, m)=\frac{4 \pi \varepsilon_{0}}{e^{2} m_{e}}\left(\frac{h}{2 \pi}\right)^{2} \frac{1}{Z}\left(\frac{1}{n^{2}}-\frac{1}{m^{2}}\right)^{-1} .
$$

Comparing the presented equation with the traditional Bohr radius formula in the form (6), we can state that they are equivalent for $m$, tending to infinity. It is indisputable that the situation under consideration, i.e. the possibility of such stable electron orbit, can take place only in the complete physical absence of radiation friction. However, the electrons of a real hydrogen-like system located in a hypothetical stationary state $r_{0}(1, \infty)$, described by the Rutherford's force ratio (5) or its energy analog in the form (23), must be slowed down and attracted by the atomic nucleus. In addition, the potential transition of electrons to an excited state $r_{h}(n, m)$ is objectively caused by their acceleration to the minimum value $v_{h}$ of the second orbital velocity.

\section{The Systematic Continuous-Deterministic Model}

Taking into account the above theoretical considerations, we consider the corresponding variation of the continuously-deterministic derivation of the parametric formula for the radii of stationary electronic orbits, based on the value of the second orbital velocity $\frac{1}{2} T_{e}\left(v_{h}\right)=$ $E\left(r_{h}, v_{h}\right)=P_{e}\left(r_{h}\right)$.

The first part of the condition under consideration, i.e. an expression of the form:

$$
\frac{m_{e} v_{h}^{2}}{2}=n h \frac{v_{h}}{2 \pi r_{h}} ;
$$

gives the corresponding intermediate result:

$$
v_{h}=\frac{n h}{\pi r_{h} m_{e}} .
$$

In turn, the basic component of the condition for the existence of a stable orbit of radius $r_{h}$, which is characteristic of the value of the electron's second orbital velocity, described by the relation (25), generates the equation:

$$
\frac{m_{e}}{2}\left(\frac{n h}{\pi r_{h} m_{e}}\right)^{2}=\frac{Z e^{2}}{4 \pi \varepsilon_{0} r_{h}}\left(1-\frac{n^{2}}{m^{2}}\right) .
$$

Thus, the formula for radii for a set of stationary electron orbits, reasoned by quantum parameters $n$ and $m$, takes the form:

$$
r_{h}(n, m)=\frac{2 r_{B}}{Z}\left(\frac{1}{n^{2}}-\frac{1}{m^{2}}\right)^{-1} .
$$

Substituting in expression (28) the values $n$ and $m$ corresponding to the spectral series of Lyman $(n=$ $1 ; m(1)=2,3,4,5,6)$, Balmer $(n=2 ; m(2)=$ $3,4,5,6)$ and Paschen $(n=3 ; m(3)=4,5,6)$, it becomes possible to obtain an alternative visualization of the energy electronic levels of hydrogen (Figure 4).

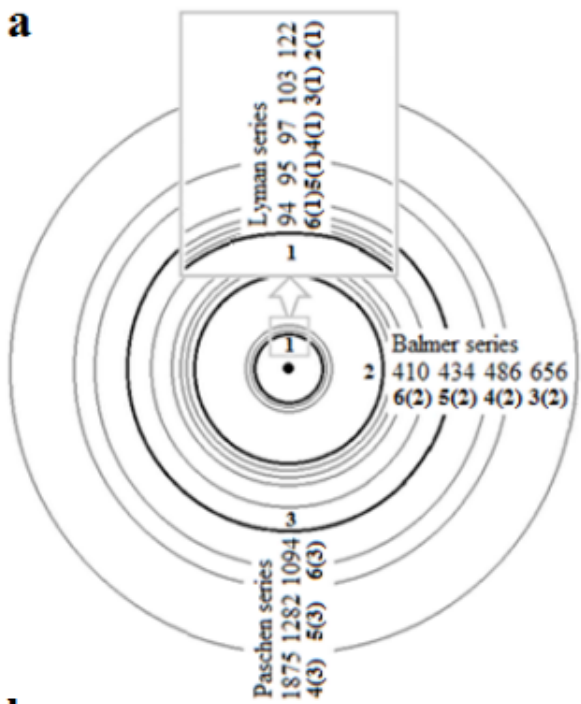

b

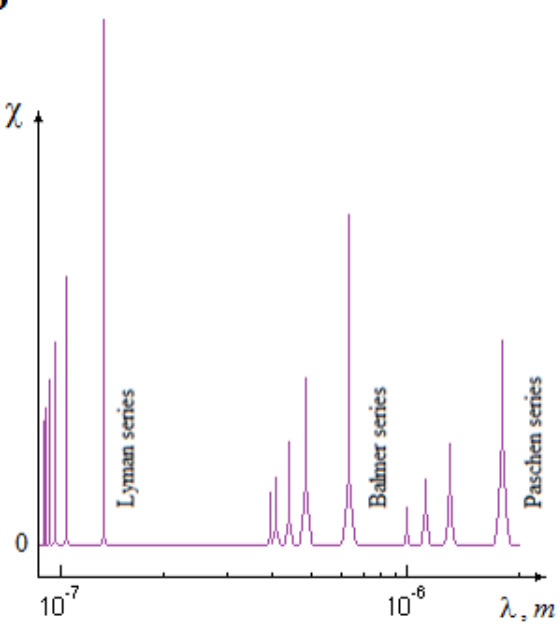

4.png

Figure 4. An alternative model of the electronic levels of the hydrogen atom. a) the scheme of the arrangement of successive electronic orbits. b) the schematic continuous graph of the long-wave dependence of optical absorption generating the Lyman, Balmer, and Paschen series. 
The physical interpretation of the proposed model of the energy levels of hydrogen spectral lines can be formulated as follows. Basic stationary levels of electrons take place for their circular orbits corresponding to discrete values of the quantum number $n$ at $m \rightarrow \infty$, and a series of excited orbitals are placed inside neighboring basic levels. In this case, the minimum value $m(n)=n+1$ corresponds to the electron orbit of the $n$ th series with the largest radius. It is obvious that the traditional (Figure 1, a) and alternative (Figure 4, a) interpretations of the spatial arrangement of the electron orbits of hydrogen are radically different from each other.

Taking into account the alternative model described above, the normal energy state of hydrogen electrons, characterized by the quantum parameters $n=1$ and $m \rightarrow \infty$, can be represented by the relations that directly follow from expressions (17) and (20):

$$
\begin{array}{r}
E_{h_{\text {norm }}}=n h f_{h \text { norm }}=h f_{\text {hnorm }} \\
P_{h \text { norm }}=\frac{Z e^{2}}{4 \pi \varepsilon_{0} r_{h}}\left(1-\frac{1^{2}}{\lim _{m \rightarrow \infty} m^{2}}\right)=\frac{Z e^{2}}{4 \pi \varepsilon_{0} r_{h}} .
\end{array}
$$

Hence, the potential-frequency condition $P_{h \text { norm }}=$ $E_{\text {hnorm }}$, which regulates the existence of excited electronic levels of a hydrogen-like system in a normal energy state, taking into account the formula of their orbital radii (28), can be represented as:

$$
\frac{Z e^{2}}{4 \pi \varepsilon_{0}} \cdot \frac{Z}{2 r_{B}}\left(\frac{1}{n^{2}}-\frac{1}{m^{2}}\right)=h f_{\text {hnorm }} .
$$

Thus, the resulting frequency equation of the studied linear spectrum, generated by continuous mathematical transformations performed in the context of the proposed approach, has the form:

$$
\begin{gathered}
f_{\text {h norm }}=Z^{2} R\left(\frac{1}{n^{2}}-\frac{1}{m^{2}}\right), \\
R=\frac{1}{h} \cdot \frac{e^{2}}{4 \pi \varepsilon_{0} 2 r_{B}}=\frac{e^{4} m_{e}}{8 \varepsilon_{0}^{2} h^{3}} .
\end{gathered}
$$

Comparison of the obtained formulation of $f_{h \text { norm }}$ with its traditional analogues shows that it is completely identical to the generalized Rydberg-Balmer's spectral formula of type (2) at $Z=1$, and structurally similar to the Moseley's x-ray law, empirically discovered in 1913:

$$
f_{r}=(Z-\sigma)^{2} R\left(\frac{1}{n^{2}}-\frac{1}{m^{2}}\right),
$$

where $\sigma-$ is the theoretical constant of shielding the atomic nucleus by electron shells, determined using the method of D. Slater [Atkins, 1974].

However, we have to state that the theoretical justification of the Rydberg's frequency constant revealed by the authors somewhat differs from its interpretation found by Bohr (11). Namely, the proposed formulation $R$ quite clearly connects the inverse value of the Planck's quantum constant and the normalized potential energy of the electrons of the hydrogen atom.

In turn, the physically observed energy outliers of quantum transitions can be directly related to the frequency resonances of attenuated harmonic oscillations by means of the well-known relation [Feynman, Leighton and Sands, 1963]:

$$
\omega_{r}=2 \pi f_{h \text { norm }}=\sqrt{\omega_{0}^{2}-2 \beta^{2}}
$$

where $\beta$ and $\omega_{0}$ - the attenuation coefficient and frequency of natural harmonic oscillations of the electronic shell.

At the same time, on the basis of repeatedly confirmed experimental data [Lide, 2009], it can be safely stated that $\beta \ll \omega_{0}$, therefore, taking into account the formula (31), the values of the natural oscillation frequencies of specific electronic orbitals of matter will be expressed as:

$$
\omega_{0}(n, m) \cong \omega_{r}=2 \pi Z^{2} R\left(\frac{1}{n^{2}}-\frac{1}{m^{2}}\right) .
$$

In addition, the studied linear spectrum considered in the context of the proposed alternative model can be interpreted as corresponding resonant modes of the optical absorption coefficient, which has the following theoretical description [Eremin and Eremina, 2016; Eremin, Eremina and Zhilindina, 2016]:

$$
\begin{array}{r}
\chi(\omega)=\sqrt{\frac{\sqrt{\varepsilon_{R e}^{2}(\omega)+\varepsilon_{I m}^{2}(\omega)}-\varepsilon_{R e}(\omega)}{2}} ; \\
\varepsilon_{R e}(\omega)=1+\frac{2}{3 \varepsilon_{0}} \alpha_{R e}(\omega) N, N=\rho / M \\
\varepsilon_{I m}(\omega)=\frac{2}{3 \varepsilon_{0}} \alpha_{I m}(\omega) N ; \\
\alpha_{R e}(\omega)=\frac{2 e^{2}}{m_{e}} \cdot \frac{\omega_{0}^{2}-\omega^{2}}{\left(\omega_{0}^{2}-\omega^{2}\right)^{2}-(2 \beta \omega)^{2}}, \\
\alpha_{I m}(\omega)=\frac{2 e^{2}}{m_{e}} \cdot \frac{2 \beta \omega}{\left(\omega_{0}^{2}-\omega^{2}\right)^{2}-(2 \beta \omega)^{2}} ;
\end{array}
$$

where $\varepsilon_{R e}(\omega)$ and $\varepsilon_{I m}(\omega)$ - real and imaginary frequency characteristics of the complex dielectric permittivity calculated using the "cybernetic model" $\varepsilon(j \omega)$; $\alpha_{R e}(\omega)$ and $\alpha_{\operatorname{Im}}(\omega)$ - classical frequency characteristics of the electronic polarizability of particles; $N$ - their volume concentrations; $\rho$ - the physical density of the sample; $M-$ the molecular mass of its formula unit.

The practical results of simulation of the long-wave electronic characteristic of hydrogen in its normal energy state, implemented using equations (34) and (35), are shown in Figure 4, b. In this case, the theoretical analysis of the appearance of the named graph provides the following physical interpretation of the intensity of the serial lines of its spectrum. 
First, the calculated analog of the frequency characteristic of the absorption coefficient of the form $\chi(\lambda)$, which corresponds to the ultraviolet Lyman series and is directly obtained for the value $\beta(1, m)=0,01 \omega_{0}(1, m)$, turns out to be adequate to the behavioral strata of the real intensities of its individual lines.

Second, the cascade decrease in the intensity of the absorption lines of the Balmer's and Paschen's series can be modeled taking into account the relative increase in the damping coefficient of the corresponding oscillation processes due to the expansion of the internal space of particles caused by their potential transitions to excited electronic levels.

\section{Modeling the Electronic Properties of Hydrogen and Helium}

Bohr's theory was a major step in the development of atomic physics and the creation of quantum mechanics. However, based, on the one hand, on the laws of classical physics, on the other - on quantum postulates, it could not explain the intensity of absorption lines, as well as the physical conditions for the implementation of certain quantum transitions. Among other things, a very serious flaw in Bohr's theory is the inability to describe the atomic spectrum of helium - the simplest of inert gases, located directly behind hydrogen in the periodic table of elements.

Figure 5 presents the results of the computational experiment aimed at deterministic modeling of the electronic properties of hydrogen, which is practically implemented using equations (28), (31), (24) and (35).

The first graph (Figure 5, a) reproduces the experimental linear spectrum of hydrogen [Lide, 2009], which is in a neutral ionized state, shown above a horizontal line, under which there are vertical segments corresponding to the values of absorption frequencies calculated by the formula (31), completely equivalent to the use of the expression (32).

The second diagram (Figure 5, b) visualizes the linear dimensions of the spherical electron orbit of the negative hydrogen ion $H^{-}$corresponding to its stationary energy state $(n=1$ and $m \rightarrow \infty)$, theoretically found by the formula (28) and shown by a solid circular line. In addition, the dotted line displays the traditional interpretation of the size of the hydrogen atom, considered as the value of the first Bohr radius (6).

The third graph (Figure 5, c) simulates the optical refraction spectrum:

$$
n(\omega)=\sqrt{\frac{\sqrt{\varepsilon_{R e}^{2}(\omega)+\varepsilon_{I m}^{2}(\omega)}+\varepsilon_{R e}(\omega)}{2}},
$$

shown by a solid line and calculated on the basis of equations (24) and (35) for the same stationary parameters of its electronic configuration as the previous characteristic. In addition, it shows a point array that reflects the experimental measurement of the simulated characteristic [Lide, 2009].

The analysis of the total set of obtained results allows us to state that the proposed relativistic method for calculating the electronic spectrum of matter is, on the one hand, as effective as the currently recognized methods of quantum mechanical calculations. On the other hand, it turns out to be much less expensive in terms of the computing resources involved, since it is entirely based on simple continuously deterministic mathematical models.

In turn, in order to test the universality of the described approach, a complex simulation of the electronic properties of helium in a neutral ionized state was carried out, implemented through the joint use of equations (2), (31) and (32), the generalized results of which are presented in Figure 6.

Analysis of the actual totality of the presented results leads to confirmation of the circumstances of the wellknown flaw in Bohr's theory, mentioned by the authors at the beginning of this section. However, in contrast to the linear spectrum of hydrogen, which physically exists only for its neutral ionized state, helium has an additional experimental characteristic, which is the linear spectrum of the gas in state of primary ionization [Lide, 2009].

The results of a similar approach to the deterministic calculation of linear spectra of single-ionized helium are presented in Figure 7.

An objective assessment of the resulting changes in the general correspondence of the frequencies of the experimentally observed absorption bands to their theoretical definition shows that the Rydberg (2) and Moseley (32) equations are also ineffective, as in the previous case. In turn, the frequency formula (31) gives a completely acceptable result. Therefore, there is actual confirmation of the principle possibility of switching from probabilistic methods of quantum computing to deterministic descriptions of the electronic properties of matter based on general relativity.

\section{Conclusions}

First, the relativistic approach proposed by the authors, based on a critical revision of the completeness of the Copenhagen quantum concept, makes it possible to theoretically derive the original formula for calculating the radii of excited electron orbitals, as well as to more strictly substantiate the numerical value of the Rydberg frequency constant without using traditional quantum Bohr postulates.

Second, the research obtained a reasonable replication of the deterministic location of the energy levels of hydrogen-like systems, which radically changes the generally accepted probabilistic interpretation of energy transitions, traditionally interpreted as quantum jumps. At the same time, the author's model allows us to explain the intensity of linear hydrogen spectra from the standpoint of the theory of harmonic oscillations, i.e. without using the Broglie-Bohr complementarity principle. 

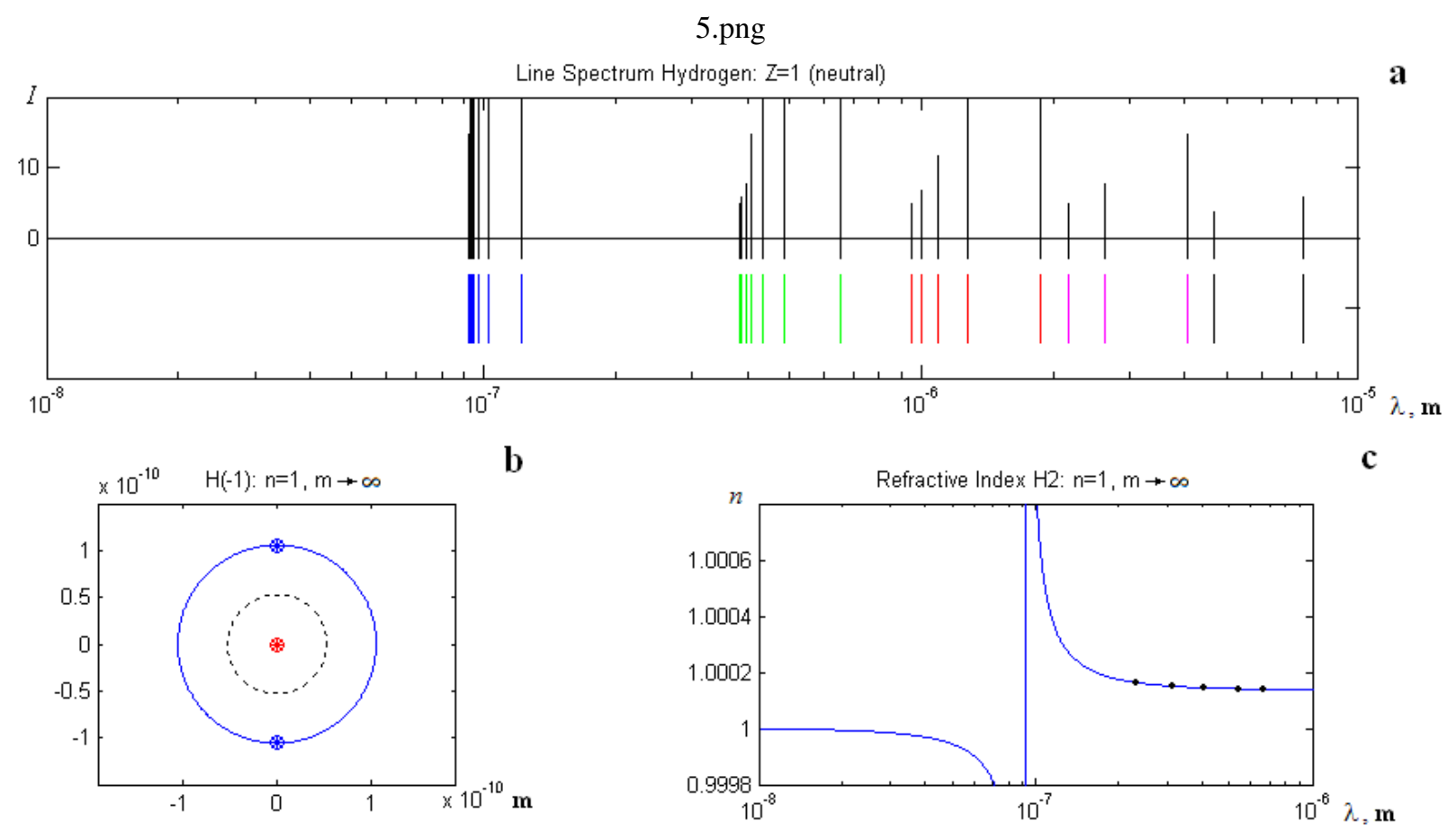

Figure 5. Results of deterministic modeling of the electronic properties of hydrogen. a) linear spectrum of radiation. b) visualization of the dimensions of a stable electron orbit. c) a graph of the long-wave dependence of the optical refractive index.
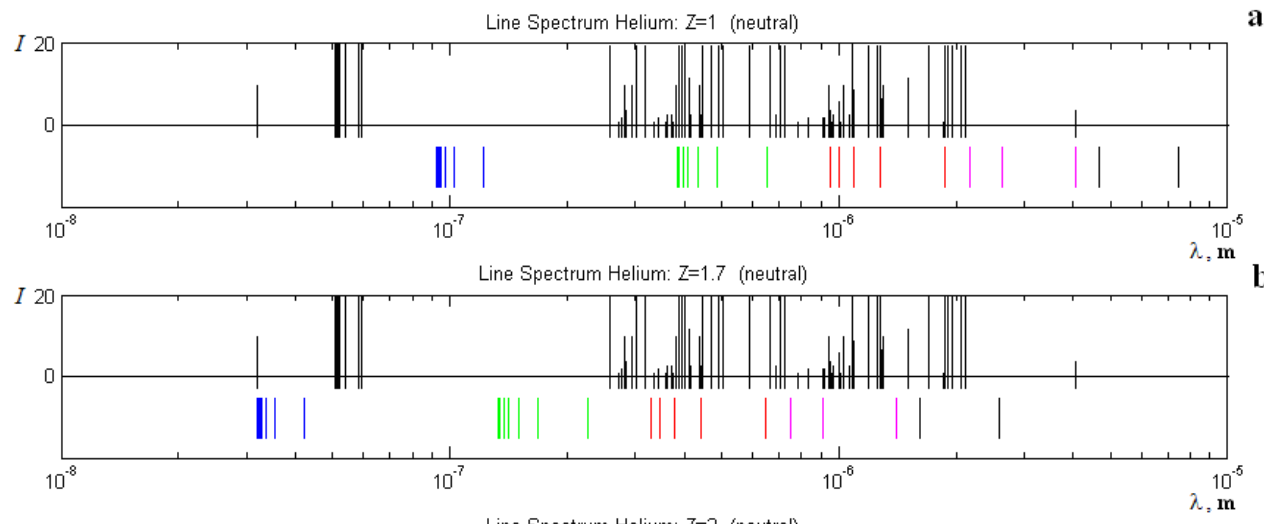

b

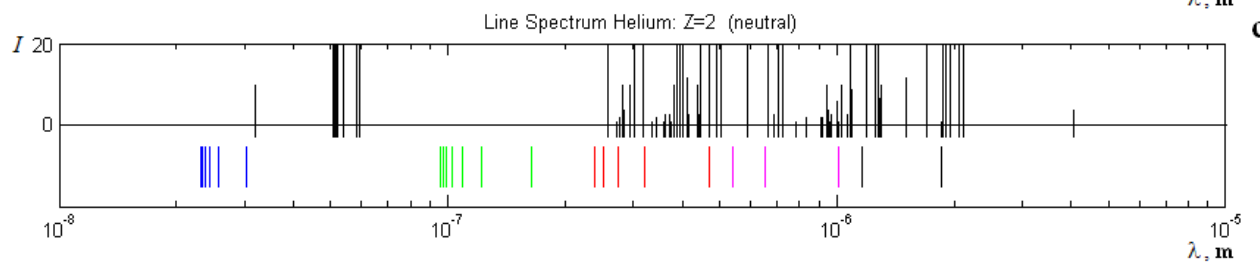

6.png

Figure 6. Comparative results of modeling the quantum properties of neutron helium. a) a linear spectrum of radiation calculated using the Rydberg equation. b) the linear spectrum of radiation calculated on the basis of Moseley's x-ray law. c) the linear spectrum of radiation calculated on the basis of the relativistic model of the form (31).

Third, the set of proposed mathematical models generates a single deterministic description of the linear spectra of neutral hydrogen and single-ionized helium, excluding probabilistic calculation methods: the Heisenberg uncertainty principle and the probabilistic interpretation of the Schrodinger-Born wave function. In addition, the formula for calculating the radii of excited elec- tron orbitals unambiguously connects the linear spectra of a substance with the geometric dimensions of its electron orbits, i.e. it provides an adequate visualization of the appearance of molecular structures.

In conclusion, we note that the presented results can be useful both for bifurcation with the optical properties of composite materials by changing their initial chemi- 

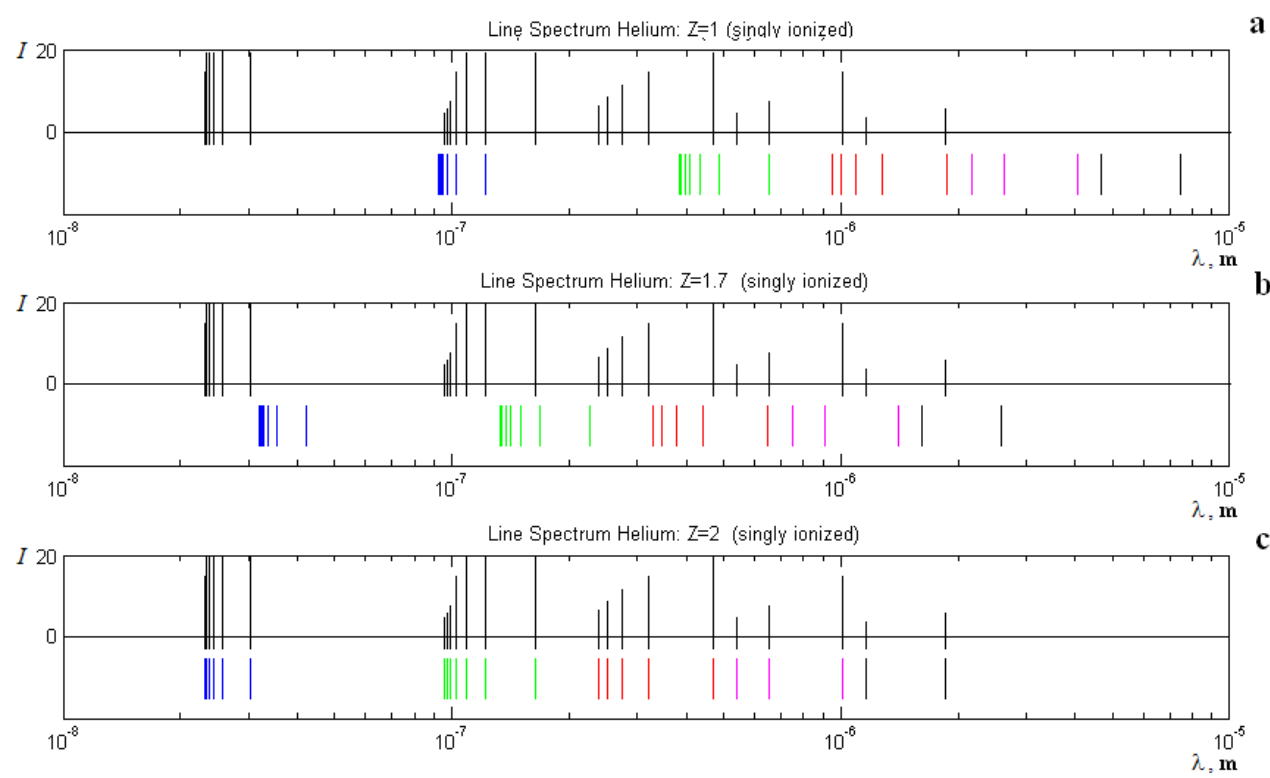

7.png

Figure 7. Comparative results of calculating the quantum properties of ionized helium. a) a linear spectrum of radiation calculated using the Rydberg equation. b) the linear spectrum of radiation calculated on the basis of Moseley's x-ray law. c) the linear spectrum of radiation calculated on the basis of the relativistic model of the form (31).

cal composition, and for identifying clear feedbacks between the energy parameters of field effects and the direct electronic responses of pure substances to them.

By the authors' opinion, the new formulation of the linear spectra of hydrogen-like systems and the model of the electronic levels of the hydrogen atom, given in this paper, in the case of their further development and extension to non-hydrogen-like systems, can change the approach to the consideration and modeling of quantum systems. In particular, to move to their deterministic representation, which will allow us to develop appropriate cybernetic models with much greater accuracy and computational efficiency compared to models based on the consideration of quantum systems as black boxes, whose input is known and with some probability the output is known.

\section{References}

Atkins, P. W. (1974). Quanta. Oxford univesity press.

Bohr, N. (1913). On the Constitution of Atoms and Molecules. Part I. In Philosophical Magazine, 26, pp. $1-24$.

Eremin, I. E. et al. (2010). Elastic Electron Polarization of Condensed Dielectrics. In Doklady Physics, 55(6), pp. 257-260.

Eremin, I. E. et al. (2014). Electronic Properties of Crystalline Fluorides of a Cubic Crystal System. In Doklady Physics, 59(1), pp. 6-9.

Eremin, I. E., Eremina, V. V. (2016). Some Cybernetic Aspects of Dielectric Systems Description. In Cybernetics and Physics, 5(4), pp. 116-122.

Eremin, I. E., Eremina, V. V., and Zhilindina, O. V.
(2016). Modeling of the Optical Spectra of M-23 Quartz Ceramicn. Glass and Ceramics, 72(11-12), pp. 417-419.

Feynman, R., Leighton, R., and Sands, M. (1963). The Feynman Lectures on Physics. Addison-Wesley Publishing Company.

Han, S., Xu, X., and Qin, F. (2018). The Unitality of Quantum B-algebras. International Journal of Theoretical Physics, 57(5), pp. 1582-1590.

Hansen, F. (2016). Quantum Entropy Derived from First Principles. Journal of Statistical Physics, 165(5), pp. 799-808.

Kobaysi, N. (2007). Introduction to Nanotechnology. Moscow: BINOM. Laboratory of Knolling's [in Russian].

Kostyukov, N. S. and Eremin, I. E. (2004). A Cybernetic Model for Simulating Elastic Electronic Polarization of a Dielectric. Electrichestvo,1, pp. 50-54 [in Russian].

Kostyukov, N. S. and Eremin, I. E. (2008). Modeling of Quartz Dielectric Spectrum in the Region of Establishing Electron Polarization Processes. Russian Physics Journal, 51(11), pp. 1149-1156.

Kumar, M. (2013). Quantum: Einstein, Bohr and the Great Debate about the Nature of Reality. Moscow: AST: CORPUS [in Russian].

Lide, D.R. (2009). CRC Handbook of Chemistry and Physics. Boca Raton.

Miao, C. et al. (2014). Remote Preparation of Quantum Entangled State in a Non-Markovian Environment. International Journal of Theoretical Physics, 53(12), pp. 4098-4106.

Nakajima, S. and Tokura, Y. (2017). Excess Entropy 
Production in Quantum System: Quantum Master Equation Approach. Journal of Statistical Physics, 169(5), pp. 902-928.

Ogata, Y. (2016). A Class of Asymmetric Gapped Hamiltonians on Quantum Spin Chains and its Characterization. Communications in Mathematical Physics, 348(3), pp. 847-895.

Robinson, T. R. and Haven, E. (2015). Quantization and Quantum-Like Phenomena: A Number Amplitude Approach Environment. International Journal of Theoretical Physics, 54(12), pp. 4576-4590.

Roldan-Charria, J. (2014). Indivisibility, Complementarity and Ontology: A Bohrian Interpretation of Quantum Mechanics. Foundations of Physics, 44(12), pp. 1336-1356.

Sych, D. and Leuchs, G. (2015). Quantum Uniqueness. Foundations of Physics, 45(12), pp. 1613-1619.

Fradkov, A.L. (2017). Horizons of cybernetical physics. Philosophical Transactions A, 375: 20160223.

Rabitz, H. (2012). Control in the sciences over vast length and time scales. Quantum Physics Letters, 1, pp. 1-19.

James, M.R. (2005). Control Theory: From Classical to Quantum Optimal, Stochastic and Robust Control: notes for Quantum Control Summer School. Australian National University, Caltech.

Doherty, A.C.et al. (2000). Quantum feedback control and classical control theory. Physical Review A, 62, 012105.

Dong, D. and Petersen, I.R. (2010). Quantum control theory and applications: a survey. IET Control Theory Applications, 4, pp. 2651-2671.

D'Alessandro, D. (2008). Introduction to quantum control and dynamics London, UK: Chapman \& Hall.

Averbukh, I. et al. (2016). Special issue on coherence and controlin the quantum world. In Journal of Physics B: Atomic, Molecular and Optical Physics, 49, 150202.

Jacobs, K. (2014). Twenty open problems in quantum control. Department of Physics, University of Massachusetts.

Youssry, A., Paz-Silva, G.A., and Ferrie, C. (2020). Characterization and control of open quantum systems beyond quantum noise spectroscopy. Nature Physics Journals: Quantum Information, 6(95). 